\title{
Sach-, Orts- und Personenregister
}

Die im Register aufgenommenen Seitenzahlen beziehen sich in der Regel auf den Text, in wenigen wichtigen Fällen auch auf die Fußnoten; auf die Anhänge wird nicht verwiesen.

10. August 1792 s. Zweite Revolution Alexandre, Georges-François Borelle dit $136,152,247$

Anjou 65

Artois 65

Aulard, Alphonse 26

Baczko, Bronislaw 19

Bailly, Edme Louis Barthélemy 248

Barbier de Tinan, Jean Jacques François Théodose 11f., 29, 33f., 36, 65, 134

Barère (de Vieuzac), Bertrand 207

Barr 190

Basel 58, 95

Bas-Poitou 65

Bataillon de l'Union 172

Baudot, Marc-Antoine $\quad 200,212,215$, $219,230,244$

Belfort 89

Berlin 59,95

Bern 136

Berr, Cerf 50,240

Berr, Marx 50ff., 55, 244

Besançon 171

Betzinger, Claude $15,27,115,120$

Bierlyn, Jean-Henri 235

Bilingualismus s. Zweisprachigkeit

Biron, Armand Louis de Gontaut, duc de Lauzun 183

Blamont 216

Blanning, Timothy 19,180

Blessig, Johann Lorenz 69

Blois 146

Blömeke, Heinrich 21

Boehmer, Georg 181

Boersch 155

Boissière, Amédée 134

Bonn 95

Bordeaux 119, 176f.

Borie, Jean 219

Boy, Adrien 200, 216

Breck, Germain 221

Brendel, François-Antoine 82

Bretagne 64
Brissot, Jacques Pierre, dit Brissot de Warville 72

Brunck de Freundeck, Jacques Pierre Simon 36, 134

Brunck de Freundeck, Richard François Philippe 34, 39f., 51f., 134, 136

Burger, Friedrich 12

Butenschoen, Friedrich 234

Caen 22

Caligostro, Graf von (Josef Balsamo) 134

Calvinisten 65

Carnot, Lazare Nicolas Maguerite 164

Carrier, Jean-Baptiste 207

Châlons-sur-Marne 195

Chamilly, Marquis de 67

Champy, Claude 109

Charente 189

Chaumas, Jean-Baptiste 155

Chaumette, Pierre Gaspard 226

Chayrou, Louis Philippe 165,170

Clauer, Carl 59, 107f., 136, 184, 221

Clavel, Etienne Pierre 203, 221

Cloots, Anarchasis 57

Cochin, Augustin 37

Colmar 48, 89

Comité de salut public s. Wohlfahrtsausschuß

Conseil général de la Commune s. Stadtrat

Conseil municipal s. Stadtrat

Cotta, Friedrich $25,58,59,62,136$, $183,184,185$

Couturier, Jean-Pierre $\quad 169,173,188$

Custine, Adam Philippe, comte de 180f., $184,186,190,240$

Dechristianisierung 13, 224-240, 253

Delâtre, A. F. 215,234

Denting 21

Dentzel, Georges Frédéric $\quad 169,173,188$

Deutsche $28,63,106,143,180,209$ $212,250 f$.

Deutschland, Krieg gegen $12,17,180$, 209 
Deutschland $12,58,60 f ., 93,106,180$

Deutschsprachige, s. Germanophone

Dièche, Antoine Claude 223

Dietrich, Friedrich $\quad 46 f ., 109,112,142$, $152,156,164,166,167-168,171,179$

Disnardy, Joseph 165

Dordogne 189

Dorsch, Anton $59,136,181 \mathrm{ff}$.

Doubs 189

Dufort, François Thomas Galbaud dit 77,134

Dumas, Mathieu 81

Dumont, André 226

Dumont, Franz 180

Dumouriez, Charles-François du Perrier, dit 190

Eckbolsheim 193f.

Edelmann, Geoffroi Louis 137

Ehrmann, Jean François 135

Eidverweigerer $27,66,76-83,91 \mathrm{f}$, 101f., 106, 143, 198

Engel, Philipp Jacob 85,86

England 211

Eymar, Jean-François-Ange d' 77

Flandern 65

Flucht des Königs $\quad$ 12, 27f., 93-101, 118, 198,252

Föderalismus 20, 22, 163, 171-180, 207

Föderalistische Revolte s. Föderalismus

Foissey, Joseph Ignace 81

Forster, Georg 185

Fouché, Joseph 226

Foussedoire, André 245f., 248

Franken 95

Frankophone 13, 69, 219, 251, 254

Französischsprachige s. Frankophone

Frey, Emanuel 156

Frey, Junius 156

Furet, François 17ff., 24, 37, 249

Gard 65

Germanophone 13f., 69, 209-224, 251, 254

Gesellschaften, affiliierte 153,164

Gintzrot, Christian 137

Gironde 12

Gloutier, Pierre Alexis 37f.

Goethe, Johann Wolfgang von 70,135

Gottekien 213

Göttingen 135

Gough, Hugh 15,122

Goujon, Jean Marie Claude Alexandre 226
Grégoire, Henri Abbé de 50,72f., 207

Greuhm, Frédéric Charles 136

Gross, Jean-Pierre 21

Gueniffey, Patrice 19,24

Guillotine 172f., 179, 193, 215, 246, 251

Guyardin, Louis $\quad 193,198,201 f ., 227 f f$.

Haffner, Isaac 69

Halle 135

Hampson, Norman 38

Hanson, Paul 22

Haupt, Friedrich 185

Haussmann, Nicolas 188

Haute-Vienne 189

Hébert, Jacques René 246

Heitz, Frédéric Charles 25

Hennegau 65

Hentz, Nicolas 226

Hérault de Séchelles, Marie Jean 81

Herder, Johann Gottfried 70

Hervé, Hyacinthe 165

Hesse, Charles de 171

Higonnet, Patrice 208

Hofmann, Andreas Joseph Christian 185

Hood, James 23

Horn, Jeff 20

Hubert, Wernhard 58

Huningue 89

Hunt, Lynn 20, 33

Immigranten $13,28,60 \mathrm{f}$., $95,126 \mathrm{ff}$., $134,136,253,254$

Jakobinerclub, Pariser $11,26,29,33$, $36,95,152,156$

Javogues, Claude 20

Juden $18,48 f f ., 58,63,65 f$., 240-244, 250

Jung, Jean $137,221,234$

Jung-Stilling 135

Karlsruhe 58

Katalonien 21

Katholiken 13, 18, 64ff., 90, 101-106, $113,130,133,138,225 \mathrm{ff} ., 251$

Katholische Kirche s. Katholiken

Kiechel, Johann Friedrich 165

Kienlin, Jean Christophe $136,164,174$

Koch, Christoph Willhelm $69,84,87$, 135

Konstituante s. Nationalversammlung

Konstitution s. Verfassung

Konvent s. Nationalversammlung

Korrespondenzen (Karte) 154 
Kosmopolitismus $\quad 27,48,56 \mathrm{ff}$., 66 , 106ff., 126, 146, 163, 180, 182

Kugler, Jean René $135 f$.

Kult der Vernunft 224-240

La Rochelle 178

Lachausse, Augustin Médard 165

Lacoste, Elie 190f., 212, 219, 229, 245

Lafayette, Gilbert, marquis de 109,158 , 171

Landau 190

Langres 159

Languedoc 65

Laurent, Claude-Hilaire 136

Lauth, Jean-Frédéric $173 \mathrm{f}$.

Laveaux, Charles $25,95,99,100,105$, $112,114,136,139,146,148,155 \mathrm{ff}$., $164,166,167,171,173,176,251$

Le Peletier, Ferdinand Louis Felix de Saint Fargeau 215

Lebas, Philippe François Joseph 194, 202, 215, 219, 235, 246

Legislative s. Nationalversammlung

Leipzig 135

Lenz, Jakob Michael Reinhold 70

Leorier 221

Leuchsenring, Ludwig 58

Levée de $300.000 \quad 171$

Levée en masse $190,192,210,219$

Levrault, Xavier $55,57,59,104,135$

Liebich, Philippe 173f.

Limoges 23

Loire 20

Longfellow, David 23

Louis, Jean Antoine 135f., 171, 174

Louvois, François Michel le Tellier 68

Lucas, Colin 20

Luckner, Nicolas, comte de 150

Ludwig XIV. 13, 67f., 200

Ludwig XVI. 12, 81, 93-101, 112, 143, $149,158,162,167,211$

Lyon $23,122,136,176,177,178$

Lyons, Martyn 23

Maine 65

Mainoni, Joseph Antoine 237f.

Mainz 59, 163, 180-186, 209f.

Mainzer Republik s. Mainz

Mallarmé, François René Auguste 229

Marat, Jean-Paul 215

Maribon-Montaut, Louis 188

Marseille 119, 155, 176ff.

Marx, Roland 15

Massé, Mathieu Joseph 223, 246
Massif Central 65

Mathieu, Jacques $75,83 \mathrm{f} ., 87,90$

Mathieu, Jean Michel 135, 248

Maximum 189, $192 f$.

Mayno, Jean Pierre Charles 134

McCoy, Rebecca 21, 70

McPhee, Peter 24

Metz 93f.

Meyer, Andreas $\quad 25,183 f$.

Milhaud, Edmond Jean-Baptiste

189 , 193, 198, 201f., 219, $227 \mathrm{ff}$.

Mirabeau, Gabriel Honoré Riqueti, comte de, dit 142

Molsheim 172, 251

Mommsen, Hans 16

Monet, Pierre François 13, 136, 169f., 173, 195, 213f., 219, 220-224, 228f., $235,236,247 \mathrm{f}$.

Montauban 65

Montaut, Louis Marie-Bon s. MaribonMontaut

Munizipalität s. Stadtrat

Münster, Straßburger 13, 230-244

Murbach, Abbé de 77

Narbonne-Lara, Louis, comte de $109 \mathrm{f}$.

Nationalversammlung 29f., 36, 38, 40, $45,54,56,60,63,76,82 f$., 110,138 , $146,148,152,162,164,173 f$., 187 , 207f., 227, 245, 247

Nestlin, Jean-Jacques 221

Nîmes 23, 65, 178

Noisette, Gaspard 109, 114, 137, 173

Nord (Département du) 189

Normandie 65

Oberlin, Jeremias Jakob 69, 135

Pape, Friedrich $182 \mathrm{ff}$.

Pas-de-Calais 65

Périgny, Charles Taillevis 136,152

Petion, Jérôme 95

Pfalz 190

Pflieger, Jean Adam 171

Pinelle, Marin 77

Popp, Charles 166

Priesterehe 102ff.

Prieur, Pierre Louis 164

Protestanten 13, 18, 28, 66ff., 106, 130, $133,135,177,225,251$

Protestantische Kirche s. Protestanten

Pyrenäen 24

Reichardt, Rolf 21 
Représentants du peuple en mission 12 , $14,20,26,28,164,169 \mathrm{ff} ., 173,187$ $205,208,210 f ., 215,217,220,226$, $235 f,, 249,252 f$.

Reuss, Rodolphe 15,228

Revolution, munizipale $20,30 \mathrm{ff}$.

Ribeauvillé 89

Richou, Louis-Joseph 249

Ritter, François Joseph 164

Rivage, Michel 136, 164

Robespierre 13, 95, 151, 239, 245f.

Rohan, Louis René Edouard, prince de $67,78 f$.

Roland de la Platière, Jean-Marie 150 , 152,166

Rouget de Lisle, Claude Joseph 12

Rousseville 217

Roussillon 208

Ruamps, Pierre Charles 188f,, 219

Rühl, Philippe Jacques $166,169,171$, $173 \mathrm{ff}$.

Sahlins, Peter 21

Sainte-Marie-aux-Mines 21

Saint-Hilaire 197f.

Saint-Just, Louis Antoine de 194ff., 202, $204,215,217,219,235,244,246$

Saint-Nicolas 94

Saint-Pierre-le-Jeune 67

Saint-Pierre-le-Vieux $\quad 67,80$

Saint-Quentin 136

Saint-Thomas 85

Saltzmann, Friedrich Rudolph 25, 45, $51,69,74,110$ f., 135,170

Saltzmann, Johann Daniel 69f., 74

Schisma, feuillantinisches $13,113 \mathrm{ff}$.

Schlettstadt 89

Schneider, Eulogius $15,25,59,95$, 100f., 104, 136, 140, 148, 171ff., 183, $210,220-224,234,242,251 \mathrm{f}$.

Schoepflin, Jean Daniel 135

Schöll, Friedrich 84f., 87, 90

Schweighäuser, Jean 135

Seine-et-Marne 21

Seinguerlet, Eugène 15

Simon, Jean Frédéric $25,137,159,164$, 217

Simond, Philibert $\quad 136,141,152,164$, 166

Soubrany de Bénistant, Pierre Amable 188

Speyer 183
Stadtrat $46,54,55,81,88,93,111,138$, $152,159,161-170,193,195,235 \mathrm{ff}$., 238,245

Stamm, Daniel $137,181,213$

Sturm auf das Straßburger Rathaus 11, $31 \mathrm{f}$.

Stuttgart 58f., 95

Sundgau 53

Sydenham, Michael 11

Tackett, Timothy 23

Taffin, Charles 136

Téterel, Antoine $172,174,248$

Thermidor 16, 245

Thomassin, Michel $166,173,187$

Tilly, Charles 24

Toulon 177f.

Toulouse 23

Tuileriensturm s. Zweite Revolution

Turckheim, Johann Friedrich von 69

Uffholz 242

Ulbrich, Claudia 21

Ulrich, Andreas 25f., 74f., 135

Valmy 180

Vendée $20,65,171,207,217$

Verdun 93

Verfassung $34,42,62 f ., 94,160,162$

Vergniaud, Pierre Victurnien 101

Verschwörung 28, 92, 197-200

Voigt, Johann Friedrich 221

Volksvertreter auf (Mission) s. Représentants du peuple (en mission)

Wahlen 32, 45f., 86, 166, 168

Wahnich, Sophie 208

Wedekind, Georg 59,181, 185, 235

Weissenburg 190, 193, 204

Wohlfahrtsausschuß 190f., 193f., 212, 238

Wolff, Hans Daniel 221

Worms 183

Wurmser, Dagobert Sigismond, comte de 204

Young, Arthur 11

Zivilverfassung des Klerus $13,64,66$, 76-92, 95, 10lf., 198, 250f.

Zweisprachigkeit $27,13,66,71 \mathrm{ff} ., 250$

Zweite Revolution $12,158,161-165$ 